\title{
TIME-RESOLVED AND NEAR-FIELD SCANNING OPTICAL MICROSCOPY STUDY ON PORPHYRIN J-AGGREGATE*
}

\author{
A. Miura, K. Matsumura, X. Su and N. Tamai \\ Department of Chemistry, School of Science, Kwansei Gakuin University \\ 1-1-155 Uegahara, Nishinomiya 662-8501, Japan
}

(Received July 23, 1998)

\begin{abstract}
Water-soluble porphyrin, 5,10,15,20-tetraphenyl-21H,23H-porphinetetrasulfonic acid (TPPS), forms J-aggregate in aqueous solution depending on experimental conditions such as $\mathrm{pH}$, dye concentration, and/or ionic strength. The steady-state fluorescence and picosecond single-photon timing spectroscopy were applied for protonated monomer and J-aggregate in aqueous solution and in thin films to reveal the dynamics in the $S_{2}$ and $S_{1}$ states. The $S_{2}$ fluorescence spectra from the protonated monomer and J-aggregate were observed in addition to the normal $S_{1}$ fluorescence. The lifetime of the $S_{2}$ state was estimated to be $\approx 5 \mathrm{ps}$ for J-aggregate, whereas the lifetime is shorter than 1 ps for protonated TPPS monomer. The mesoscopic structures of J-aggregate in thin film with and without polymer on the glass surface were examined by scanning near-field optical microscopy. With the surface topography and scanning near-field optical microscopy transmission images, TPPS J-aggregate was found to form a long and narrow tube-like structure which has a few $\mu \mathrm{m}$ length, $0.2-0.5 \mu \mathrm{m}$ width, and $5-30 \mathrm{~nm}$ height. An unidirectional orientation of the structure was also found, which may be originated from the spin-coating process.
\end{abstract}

PACS numbers: $78.47 .+p$

\section{Introduction}

Recently, much attention has been focused on the dynamics and spectroscopy in small domains [1]. One of the most fruitful optical spectroscopic technique in small domains is scanning near-field optical microscopy (SNOM), which provides a spatial resolution better than the diffraction limit $(\lambda / 2)$ for imaging and spectroscopy applications [2-16]. Molecular aggregates and microcrystals have been

*The results of this paper were initially presented at The Jabtonski Centennial Conference on Luminescence and Photophysics, July 23-27, 1998, Torun', Poland. 
the subject of SNOM study because of their heterogeneous properties on the solid surfaces. J-aggregates of pseudoisocyanine (PIC) dyes have been widely investigated by Barbara and coworkers using fluorescence, time-resolved fluorescence, and polarization SNOM techniques [11-15]. They found that J-aggregate of PIC takes on a number of different shapes such as long, fibrous structures that can be randomly oriented and highly curved on micron and larger distance scales. In addition, the extent of excitation migration along J-aggregate was estimated to be less than $\approx 50 \mathrm{~nm}$ by the analysis of photobleaching induced by the light of SNOM tip. The formation of an interesting microstructure such as ring-shaped assemblies has been also reported using a platinum porphyrin derivative in the evaporation of solutions on a substrate [16].

It is also known that some porphyrin derivatives form J-aggregates $[17,18]$. The aggregates of porphyrins play a dominant role in nature such as photosynthetic systems [19]. Water-soluble porphyrin, 5,10,15,20-tetraphenyl-21H,23H-porphinetetrasulfonic acid (TPPS), is known to form J-aggregate in aqueous media depending upon experimental conditions such as $\mathrm{pH}$, dye concentration and/or ionic strength [18]. The mesoscopic structure of porphyrin J-aggregates as well as their dynamics in the $S_{2}$ state, however, are not well known until now. In the present study, we have examined the fluorescence properties of TPPS J-aggregate in the $S_{2}$ state as well as protonated monomer of TPPS by picosecond single-photon timing spectroscopy. Thin films of TPPS were also prepared by the spin coating of aqueous solution with and without polymer on the glass plates. The mesoscopic structure of J-aggregate of TPPS in thin films has been investigated by SNOM. The surface topography and SNOM transmission images for thin films were compared with each other.

\section{Experimental}

5,10,15,20-tetraphenyl-21H,23H-porphinetetrasulfonic acid was purchased from Tokyo Kasei Co. and used without further purification. The polymer solution of polyvinyl sulfate (PVS, Wako Chemical Co., colloidal titration grade, degree of polymerization $\approx 1500$ ) was used for the preparation of thin films. The water was purified to the resistivity of $\approx 18 \mathrm{M} \Omega \mathrm{cm}$ by a water purification system (Yamato Co., Millipore WQ500). By dissolving a certain amount of PVS in purified water, the solution was heated to $70^{\circ} \mathrm{C}$ and then cooled to the room temperature. TPPS aqueous solution was added to the PVS solution, which was adjusted to the $\mathrm{pH}$ value of 2.2-0.7 by adding the concentrated $\mathrm{HCl}$ (Wako Chemical Co., superspecial grade 35\%). A pH meter (Shibata Science Instruments Co., digital pH meter 671 ) was used to measure the $\mathrm{pH}$ value of solution. The sample was spin coated on microscope glass plates (Matsunami, thickness $\approx 150 \mu \mathrm{m}$ ).

Steady-state fluorescence and excitation spectra, and absorption spectra were measured by a fluorescence (Instruments SA, SPEX FluroMax 2) and absorption (Hitachi, U-3210) spectrophotometers, respectively. Time-resolved fluorescence decay curves were detected by picosecond single-photon timing spectroscopy, the details of which were written elsewhere [20]. Briefly, the sample was excited at $\approx 360 \mathrm{~nm}$ of second harmonics of a synchronously-pumped, mode-locked femtosecond dye laser, in which the repetition rate was reduced by an external 
pulse picker. Depending on the experimental conditions, the instrument response function of the system was $30-50$ ps by using a microchannel-plate photomultiplier (R2809U). A non-linear least-squares iterative convolution method based on a Marquardt algorithm was used for the decay curve analysis [21].

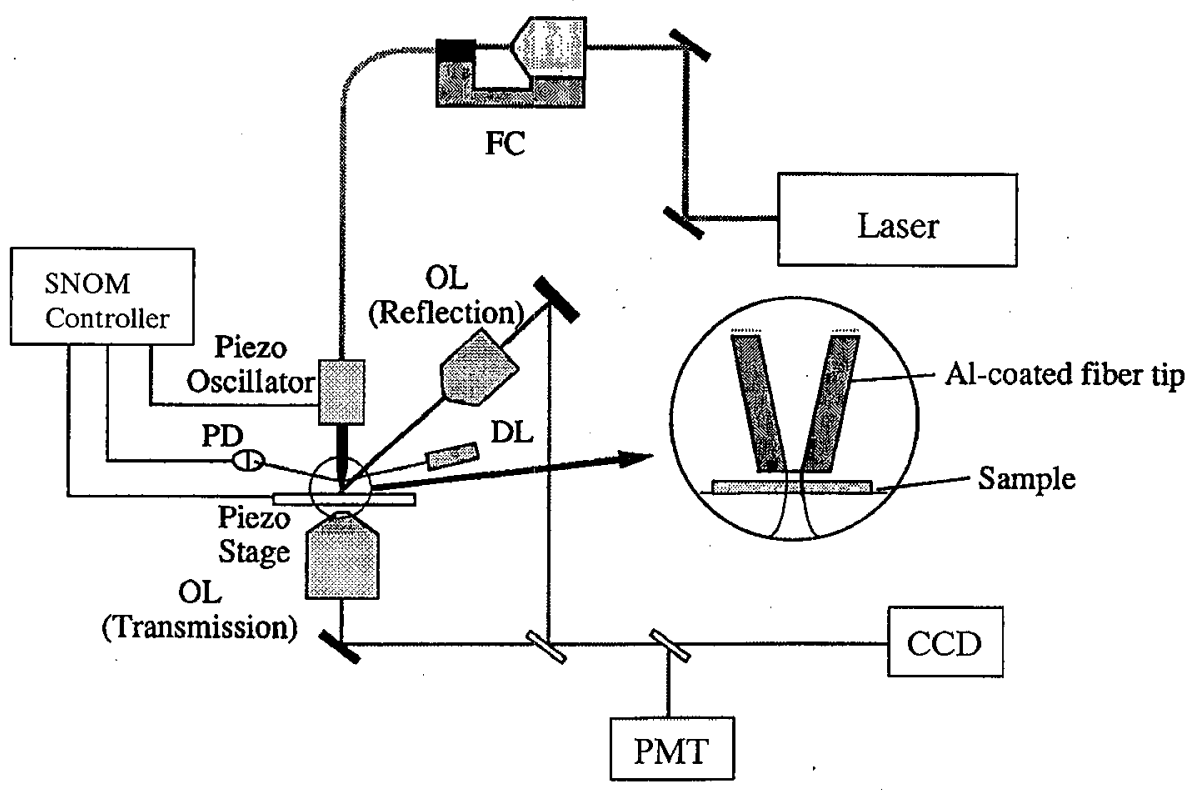

Fig. 1. A block diagram of the SNOM system. $\mathrm{CW} \mathrm{Ar}{ }^{+}$laser $(488 \mathrm{~nm})$ or $\mathrm{Nd}: \mathrm{YVO}_{4}$ laser $(532 \mathrm{~nm})$ was coupled to the cleaved end of the fiber. FC - fiber coupler, PD photodiode, OL - objective lens, DL - diode laser, PMT - photomultiplier.

The SNOM instrument was purchased from Topometrix (Aurora) in which the shear-force feedback mechanism was used to control the tip-sample separation as illustrated in Fig. 1. An Al-coated fiber tip with an aperture diameter of 50-100 $\mathrm{nm}$ was used to measure the surface topography and the transmission images. A CW Ar ${ }^{+}$laser (488 nm, Spectra Physics, 161C) or $\mathrm{Nd}: \mathrm{YVO}_{4}$ laser (532 $\mathrm{nm}$, Spectra Physics, Millennia) of $\approx 1 \mathrm{~mW}$ was coupled into the cleaved end of the fiber. Transmission image was obtained by a photomultiplier as a function of sample position.

\section{Results and discussion}

The absorption spectra of TPPS in solution are illustrated in Fig. 2. As clearly shown in the figure, absorption spectra of TPPS strongly depend on the $\mathrm{pH}$ of the solution [18]. At higher $\mathrm{pH}$ region, TPPS shows main absorption peaks at $410 \mathrm{~nm}$ and $633 \mathrm{~nm}$, corresponding to Soret- and Q-bands of the monomer, respectively. At $\mathrm{pH}$ less than 4 , TPPS is protonated and forms TPPS dianion as illustrated in Scheme I, which shows main absorption peaks at $434 \mathrm{~nm}$ (Soret-band) 

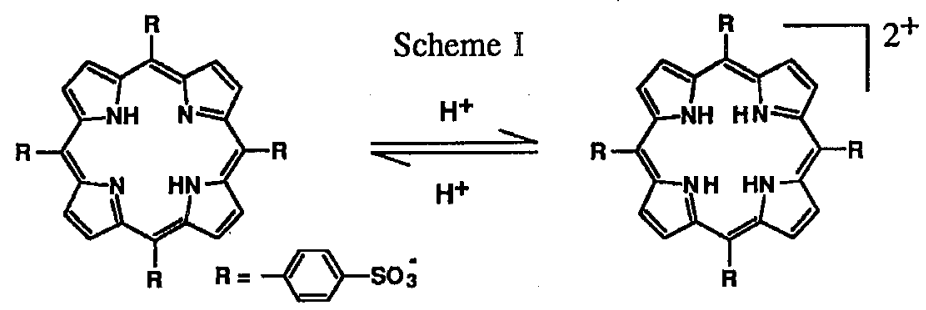

and $644 \mathrm{~nm}$ (Q-band). As the $\mathrm{pH}$ value is decreased further $(\mathrm{pH}<2.2)$, new absorption peaks appear at $490 \mathrm{~nm}$ and $706 \mathrm{~nm}$, together with a decrease in intensity of Soret-band at $434 \mathrm{~nm}$ and Q-band at $644 \mathrm{~nm}$. The band width of the new absorption peak at $490 \mathrm{~nm}$ is $\approx 8 \mathrm{~nm}\left(\approx 333 \mathrm{~cm}^{-1}\right)$ which is narrower than that of the monomer. This sharp Soret-band is characteristic of J-aggregate of TPPS $[18,22,23]$.

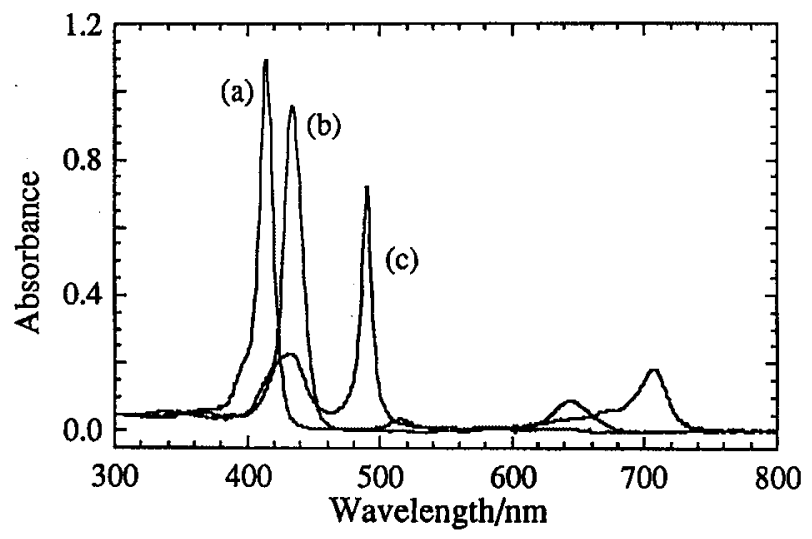

Fig. 2. Absorption spectra of TPPS $\left(1 \times 10^{-5} \mathrm{M}\right)$ in aqueous solution (2 mm cell) at various $\mathrm{pH}$ conditions: (a) $\mathrm{pH}=10.5$, (b) $\mathrm{pH}=2.7$, (c) $\mathrm{pH}=0.8$.

The J-aggregate of TPPS was also formed in polymer thin film, which was confirmed by absorption spectrum as illustrated in Fig. 3 . The characteristic absorption band at $490 \mathrm{~nm}$ was clearly observed although the band width was larger than that in aqueous solution and was $\approx 20 \mathrm{~nm}\left(\approx 830 \mathrm{~cm}^{-1}\right)$. This may be originated from the inhomogeneous broadening in the thin polymer film [24]. This is in contrast with the $\mathbf{J}$-aggregate of $1,1^{\prime}$-diethyl-2, $2^{\prime}$-cyanide iodide (PIC) which has similar position and width for the bulk solution and near-field fluorescence spectra indicating the little inhomogeneous broadening of the PIC J-aggregate in polymer thin film [11]. For the comparison with PIC J-aggregate, further analysis of absorption and fluorescence by SNOM is indispensable.

Figure 4 illustrates fluorescence spectra of TPPS at various $\mathrm{pH}$ conditions. When the sample with $\mathrm{pH}=3.8$ was excited to the higher excited state $\left(S_{2}\right)$, fluorescence spectrum with a maximum at $\approx 445 \mathrm{~nm}$ was observed in addition to the 


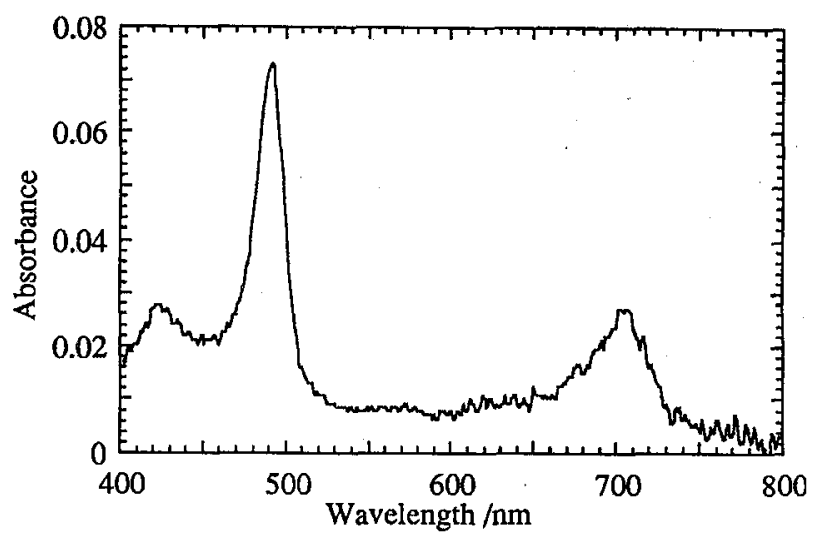

Fig. 3. Absorption spectra of TPPS/PVS thin film. The film was prepared by a spin coating of the solution containing $5 \times 10^{-4} \mathrm{M}$ of TPPS and $5 \mathrm{mg}$ of PVS in $5 \mathrm{ml}$ of purified water on a cover glass plate.

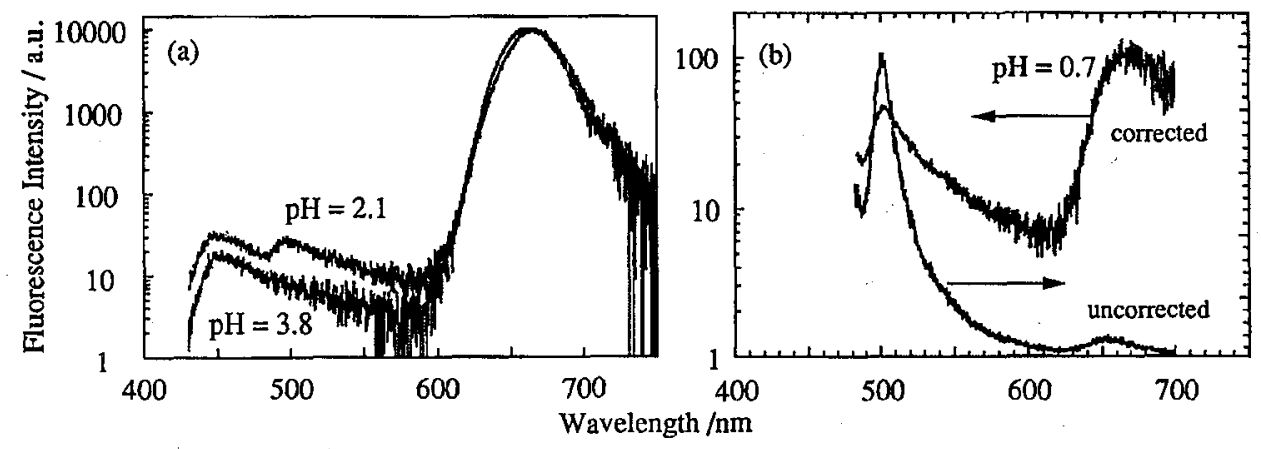

Fig. 4. (a) Corrected fluorescence spectra (in arbitrary units) of TPPS $\left(1 \times 10^{-4} \mathrm{M}\right)$ at $\mathrm{pH}=3.8$ and 2.1 , excited at $360 \mathrm{~nm}$. (b) Corrected and uncorrected fluorescence spectra of TPPS $\left(2 \times 10^{-4} \mathrm{M}\right)$ at $\mathrm{pH}=0.7$, excited at $400 \mathrm{~nm}$.

$S_{1}$ fluorescence with a peak at $\approx 670 \mathrm{~nm}$ which is comparable to the reported value of $680 \mathrm{~nm}$ for protonated TPPS monomer [22]. The shorter wavelength emission is probably assigned to the $S_{2}$ fluorescence of the protonated TPPS monomer. The intensity of the $S_{2}$ fluorescence is three orders of magnitude smaller than the $S_{1}$ fluorescence. This suggests that the fluorescence quantum yield of the $S_{2}$ state is of the order of $10^{-4}$. As the $\mathrm{pH}$ was reduced, a new fluorescence band appears at $\approx 500 \mathrm{~nm}$, which may be originated from the $S_{2}$ state of J-aggregate. This becomes clear when the $\mathrm{pH}$ is reduced to 0.7 (Fig. $4 \mathrm{~b}$ ), in which the fluorescence from the shorter wavelength at $\approx 500 \mathrm{~nm}$ is dominant as compared with that of the higher $\mathrm{pH}$. The Stokes shift in the $S_{2}$ state of TPPS is very small and has a value of $570 \mathrm{~cm}^{-1}$ for protonated TPPS monomer and $400 \mathrm{~cm}^{-1}$ for J-aggregate. This value is comparable to other porphyrin compounds such as zinc tetraphenylporphin (ZnTPP) [25]. 
For the analysis of fluorescence properties, fluorescence decay curves at various conditions were measured in solution and films. The fluorescence decay of the $S_{1}$ state of protonated TPPS observed at $\approx 675 \mathrm{~nm}$ is illustrated in Fig. 5. The decay curve was analyzed by a single-exponential decay function with a lifetime of $3.95 \mathrm{~ns}$. This value is in good agreement with the reported value of $3.87 \mathrm{~ns}$ for protonated TPPS [22].

Figure 6 shows fluorescence decays observed at $460 \mathrm{~nm}$ and $500 \mathrm{~nm}$ corresponding to that of the $S_{2}$ state of protonated monomer and J-aggregate, respec-

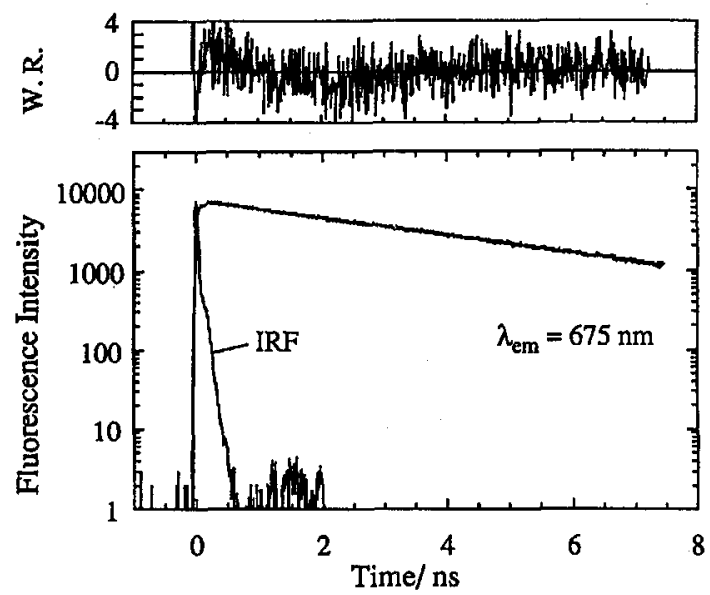

Fig. 5. Fluorescence decay curve (in arbitrary units) of protonated TPPS monomer in aqueous solution $(\mathrm{pH}=3.75)$ excited at $\approx 360 \mathrm{~nm}$ and monitored at $\approx 675 \mathrm{~nm}$. The weighted residual was given in the upper part. IRF is the system response function.
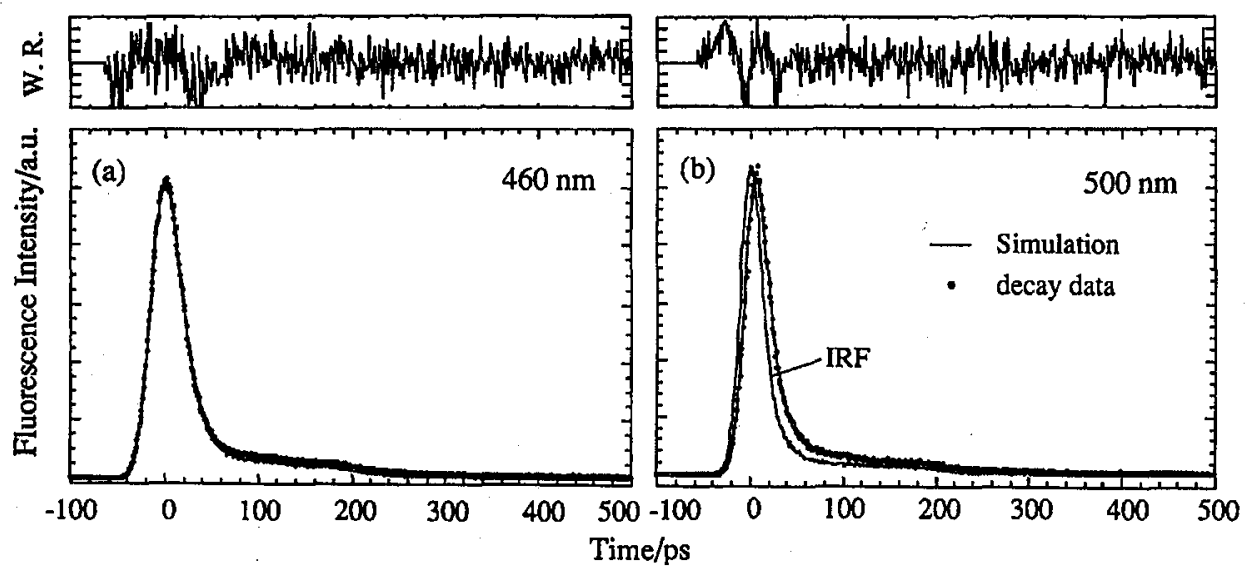

Fig. 6. Fluorescence decay curves (in arbitrary units) of protonated TPPS monomer with a $\mathrm{pH}$ of 3.75 observed at $460 \mathrm{~nm}$ (a) and TPPS J-aggregate with a $\mathrm{pH}$ of 0.84 observed at $500 \mathrm{~nm}(\mathrm{~b})$ in aqueous solution. The samples were excited at $\approx 360 \mathrm{~nm}$. 
tively. As clearly shown in the figure, very fast decay component with a lifetime shorter than 10 ps is dominant in both fluorescence decays. It was found that from the deconvolution procedure the lifetime of protonated TPPS monomer was $<1 \mathrm{ps}$ (amplitude $>99.9 \%$ ) and that of the J-aggregate was $\approx 5$ ps (amplitude $98.5 \%$ ). A similar $S_{2}$ fluorescence behavior was observed in polymer thin film containing TPPS J-aggregate. The lifetime of the $S_{2}$ state was estimated to be $\approx 6 \mathrm{ps}$, which is comparable to that of the $\mathrm{J}$-aggregate in aqueous solution. Up to now, only a few direct measurements of the lifetime of $S_{2}$ state have been reported by using Streak camera and fluorescence up-conversion method [25-27]. The $S_{2}$ lifetimes of $4.5 \mathrm{ps}$ and $<2.5 \mathrm{ps}$ were estimated for ruthenium and cadmium tetrabenzoporphyrins [26]. The $S_{2}$ lifetimes of ZnTPP in acetonitrile, dichloromethane, and ethanol were $3.5 \mathrm{ps}, 0.75 \mathrm{ps}$, and $2.35 \mathrm{ps}$, respectively [25, 27]. However, the most of the study has been focused on metalloporphyrins, and there is no direct measurement on free base porphins. The $S_{2}$ fluorescence quantum yield of free base porphin $\left(\mathrm{H}_{2} \mathrm{TPP}\right)$ has been reported to be less than $3 \times 10^{-4}$ and smaller than that of ZnTPP $\left(1 \times 10^{-3}\right)$ [28]. This result is in good agreement with the current experimental result that the protonated TPPS monomer has the lifetime shorter than $1 \mathrm{ps}$ in the $S_{2}$ state. The shorter $S_{2}$ lifetime in free base porphin has been interpreted in terms of nonradiative transition. Since the large frequency of the $\mathrm{N}-\mathrm{H}$ stretching vibration may act as an accepting mode of the electronic excitation energy from the $S_{2}$ state, the $S_{2}$ lifetime of free base porphin is very short [29]. In contrast, no central $\mathrm{N}-\mathrm{H}$ bond exists in metalloporphyrins and $\mathrm{N}$-metal bond has lower vibrational frequencies, which may be responsible for relatively larger $S_{2}$ fluorescence quantum yield and few ps lifetime in the $S_{2}$ state. On the other hand, the $S_{2}$ lifetime of J-aggregate of TPPS which has central N-H bond is longer than that of the protonated TPPS monomer. Taking into account the above discussion, another factor should be considered for the relatively longer $S_{2}$ lifetime of $\mathrm{J}$-aggregate. The delocalization of the electronic excitation energy in the $S_{2}$ state may be responsible for the longer $S_{2}$ lifetime. However, the detailed mechanism is not well known and further investigation is necessary to understand the difference in the dynamics of the $S_{2}$ state between the protonated monomer and J-aggregate of TPPS.

For analyzing the mesoscopic structure of J-aggregate of TPPS, SNOM technique was applied for the thin film of TPPS on the glass plates. Figure 7 illustrates topographic and corresponding near-field images of spin coated TPPS thin film without polymer. The formation of J-aggregate in the thin film was confirmed by steady-state absorption spectrum. As clearly shown in the figure, topographic image shows a long and narrow rod-like structure similar to a microcrystal. The analysis of topographic image indicates that mesoscopic structures of TPPS have a few $\mu \mathrm{m}$ length and $0.2-0.5 \mu \mathrm{m}$ width. The height of this structure is $10-60 \mathrm{~nm}$. The transmission image at $488 \mathrm{~nm}$ shows a dark area corresponding to the topography. This result indicates that the rod-like structure is responsible for the J-aggregate of TPPS. A pair of rod-like structure and an unidirectional orientation of the J-aggregate were observed as clearly shown in Fig. 8. A large aggregate structure depicted in line $a$ and $c$ is an exceptional case. The line analysis of the topography in Fig. 8B shows the ordinary height distribution being in the range 


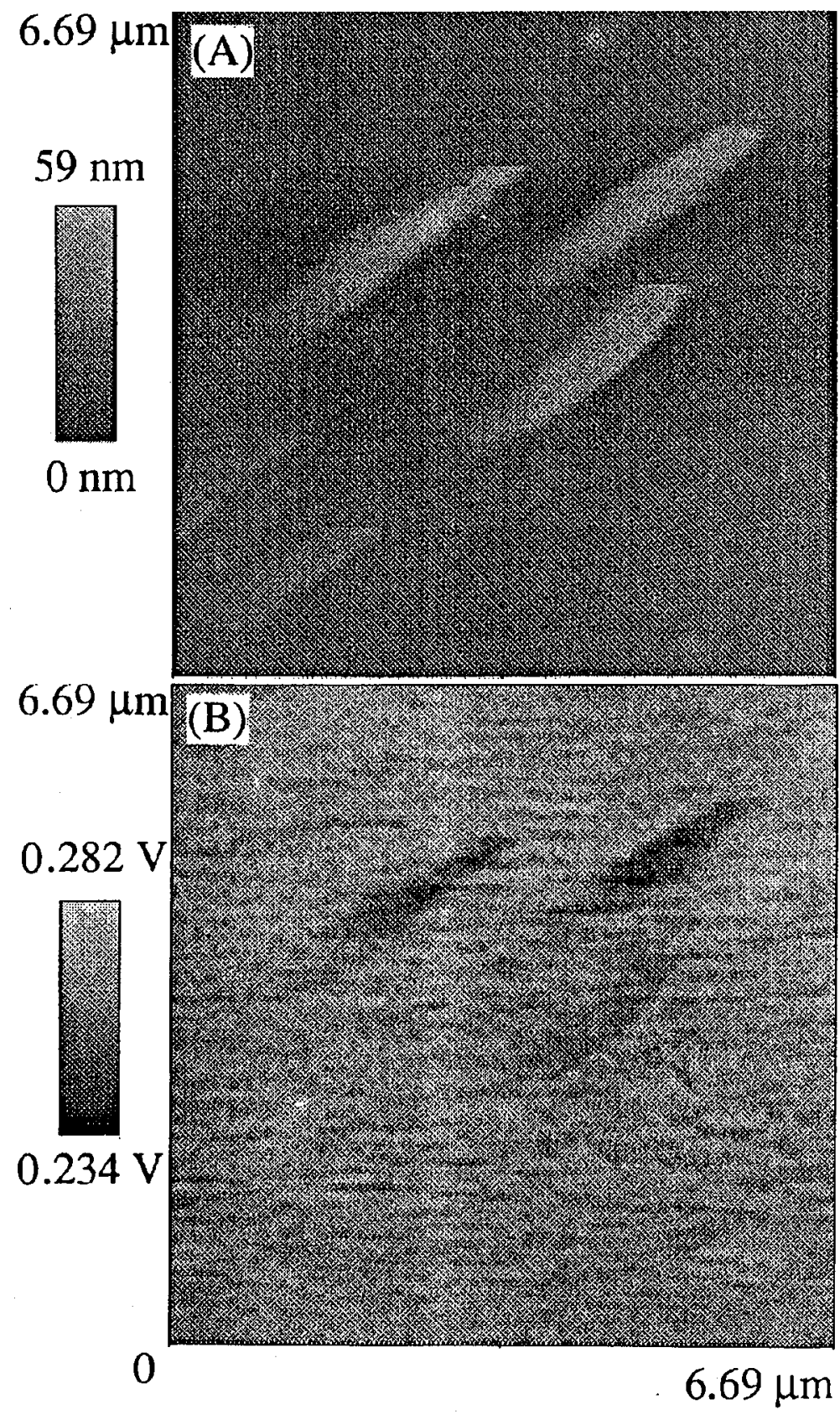

Fig. 7. (A) Topographic image and (B) corresponding SNOM transmission image of TPPS thin film without polymer. The sample was prepared by spin coating of TPPS solution with a concentration of $1 \times 10^{-4} \mathrm{M}$ and $\mathrm{pH}=1.74$ on a. microscope cover glass plate. Transmission image was obtained by $\mathrm{Ar}^{+}$laser at $488 \mathrm{~nm}$. 


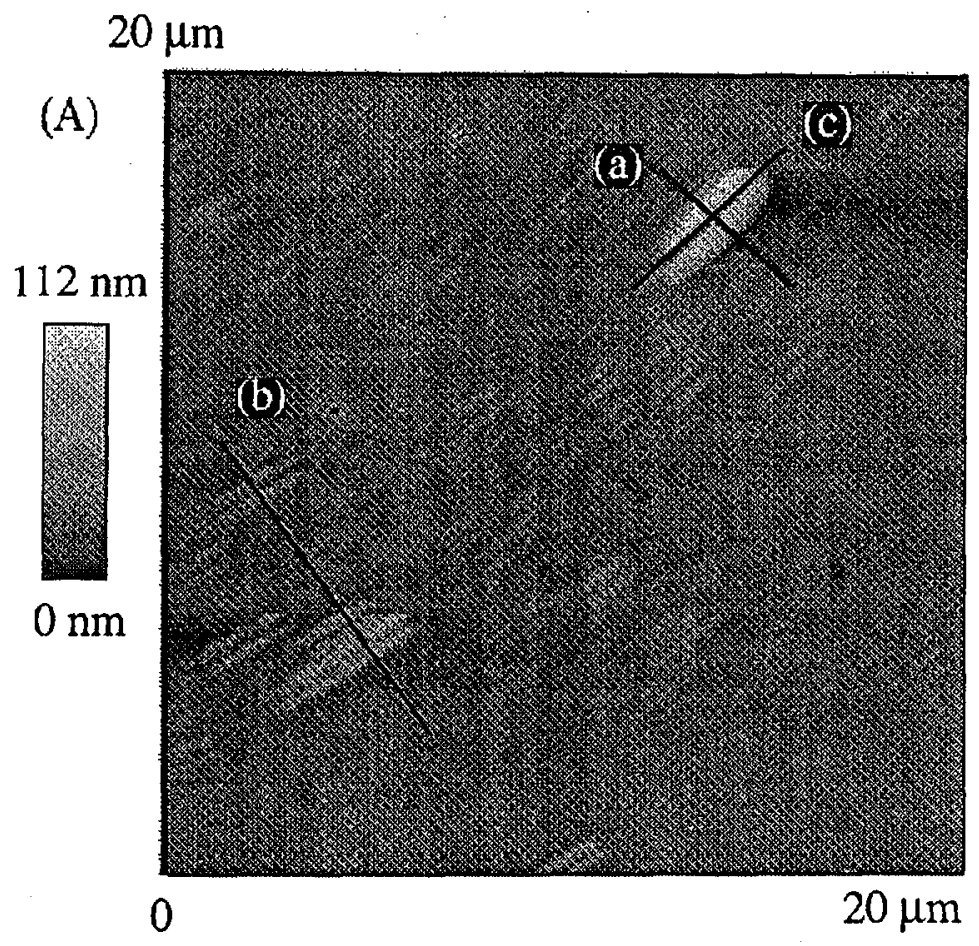

(B)

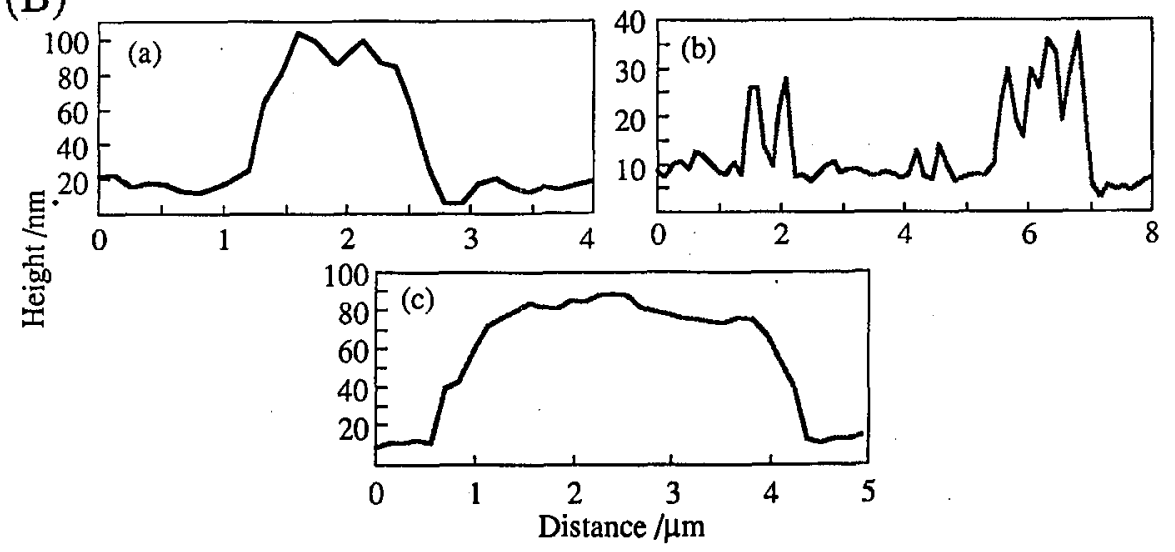

Fig. 8. (A) Topographic image of TPPS thin film without polymer and (B) line analysis of the topography for lines $a, b$, and $c$. The sample is the same as in Fig. 7 .

from 5-30 $\mathrm{nm}$ (line $b$ ). The unidirectional orientation of the structure may be originated from the spin-coating process in which the solution was dropped on a rotating glass plate. 


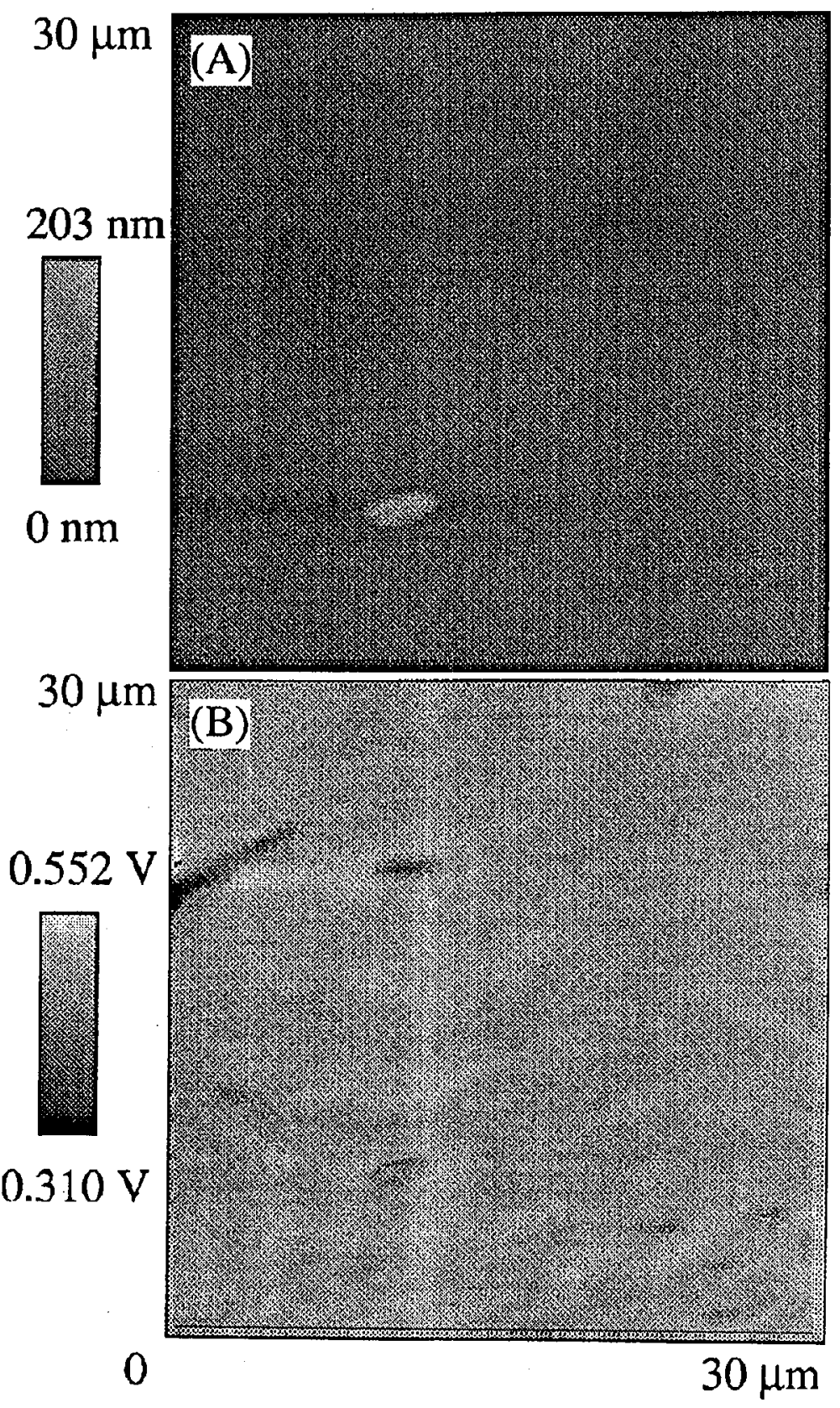

Fig. 9. (A) Topographic image and (B) corresponding SNOM transmission image of TPPS/PVS thin polymer film. The sample was prepared by spin coating of TPPS/PVS solution with concentrations of $2.5 \times 10^{-5} \mathrm{M}$ for TPPS and $12 \mathrm{mg} / \mathrm{ml}$ for PVS. The $\mathrm{pH}$ of the sample was adjusted to be 2.2 . 
For the polymer thin film of TPPS J-aggregate, the transmission or fluorescence SNOM image of the film will be of great importance for analyzing the mesoscopic structure, because the structure with a typical height distribution of 5-30 nm may be concealed by the polymer film. For the convenience, the topography and SNOM transmission images of TPPS/PVS thin film were measured and illustrated in Fig. 9. The height distribution of polymer itself is rather smooth, and thus the structure of J-aggregate of TPPS is not so clear as in Figs. 7 and 8 for the sample without polymer. However, the transmission SNOM image is clearer than the topography, and rod-like structure similar to that observed in the thin film without polymer is observed. The mesoscopic structure of TPPS J-aggregate observed in the present study is different from that observed for PIC J-aggregate, in which the long needle- or fiber-like structure was observed [11-15].

In conclusion, fluorescence dynamics of J-aggregate and protonated monomer of water-soluble porphyrin, 5,10,15,20-tetraphenyl-21H,23H-porphinetetrasulfonic acid, was investigated by picosecond single-photon timing spectroscopy. The $S_{2}$ fluorescence in addition to the normal $S_{1}$ fluorescence was observed for protonated monomer and J-aggregate. The lifetimes of the $S_{2}$ state of protonated monomer and $\mathrm{J}$-aggregate in aqueous solution were estimated to be $<1 \mathrm{ps}$ and $\approx 5 \mathrm{ps}$, respectively. The $S_{2}$ lifetime in thin polymer film of J-aggregate was similar to the value in aqueous solution. The surface topography and transmission image obtained by SNOM revealed the mesoscopic structure of the thin film of J-aggregate. A long and narrow tube-like structure which has a few $\mu \mathrm{m}$ length, $0.2-0.5 \mu \mathrm{m}$ width, and 5-30 $\mathrm{nm}$ height was found as a mesoscopic structure of J-aggregate. An unidirectional orientation, which may be originated from the spin-coating process, was also found. A further investigation of the $\mathrm{pH}$ and concentration dependence on the mesoscopic structure of J-aggregate is now in progress.

\section{Acknowledgments}

This work was partially supported by Grant-in-Aid for Scientific Research on Priority Area of Near-field Nano-optics (No. 10135226) from the Ministry of Education, Science, Sports and Culture, Japan.

\section{References}

[1] Microchemistry - Spectroscopy and Chemistry in Small Domains, Eds. H. Masuhara, F.C. De Schryver, N. Kitamura, N. Tamai, Elsevier, North-Holland, 1994.

[2] E. Betzig, J.K. Trautman, T.D. Harris, J.S. Weiner, R.L. Kostelak, Science 251, 1468 (1991).

[3] E. Betzig, J.K. Trautman, Science 257, 189 (1992).

[4] E. Betzig, R.J. Chichester, Science 262, 1422 (1993).

[5] K. Lieberman, S. Harush, A. Lewis, R. Kopelman, Science 247, 59 (1990).

[6] D. Birbaum, S.-K. Kook, R. Kopelman, J. Phys. Chem. 97, 3091 (1993).

[7] R.C. Dunn, G.R. Holton, L. Mets, X.S. Xie, J. Phys. Chem. 98, 3094 (1994).

[8] X.S. Xie, R.C. Dunn, Science 265, 361 (1994).

[9] W.P. Ambrose, P.M. Goodwin, J.C. Martin, R.A. Keller, Science 265, 364 (1994). 
[10] W.P. Ambrose, P.M. Goodwin, J.C. Martin, R.A. Keller, Phys. Rev. Lett. 72, 160 (1994).

[11] D.A. Higgins, P.F. Barbara, J. Phys. Chem. 99, 3 (1995).

[12] D.A. Higgins, P.J. Reid, P.F. Barbara, J. Phys. Chem. 100, 1174 (1996).

[13] P.J. Reid, D.A. Higgins, P.F. Barbara, J. Phys. Chem. 100, 3892 (1996).

[14] D.A.V. Bout, J. Kerimo, D.A. Higgins, P.F. Barbara, J. Phys. Chem. 100, 11843 (1996).

[15] D.A. Higgins, J. Kerimo, D.A.V. Bout, P.F. Barbara, J. Am. Chem. Soc. 118, 4049 (1996).

[16] J. Hofkins, L. Latterini, P. Vanoppen, H. Faes, K. Jeuris, S. De Feyter, J. Kerimo, P.F. Barbara, F.C. De Schryver, A.E. Rowan, R.J.M. Nolte, J. Phys. Chem. B 101, 10588 (1997).

[17] E.B. Fleisher, J.M. Palmer, T.S. Srivastava, A. Chatterjee, J. Am. Chem. Soc. 93, 3162 (1971).

[18] O. Ohno, Y. Kaizu, H. Kobayashi, J. Chem. Phys. 99, 4128 (1993).

[19] W. Kühlbrandt, Nature 374, 497 (1995).

[20] N. Tamai, M. Ishikawa, N. Kitamura, H. Masuhara, Chem. Phys. Lett. 184, 398 (1991).

[21] P.R. Bevington, Data Reduction and Error Analysis for the Physical Sciences, McGraw-Hill, New York 1969.

[22] N.C. Maiti, M. Ravikanth, S. Mazumdar, N. Periasamy, J. Phys. Chem. B 99, 17192 (1995).

[23] N.C. Maiti, S. Mazumdar, N. Periasamy, J. Phys. Chem. B 102, 1528 (1998).

[24] M. Wubs, J. Knoester, Chem. Phys. Lett. 284, 63 (1998).

[25] G.G. Gurzadyan, T.-H. Tran-Thi, T. Gustavsson, J. Chem. Phys. 108, 385 (1998).

[26] J. Aaviksoo, A. Freiberg, S. Savikhin, G.F. Stelmakh, M.P. Tsvirko, Chem. Phys. Lett. 111, 275 (1984).

[27] H. Chosrowjan, S. Taniguchi, T. Okada, S. Takagi, T. Arai, K. Tokumaru, Chem. Phys. Lett. 242, 644 (1995).

[28] S. Tobita, Y. Kaizu, H. Kobayashi, I. Tanaka, J. Chem. Phys. 81, 2962 (1984).

[29] Y. Kurabayashi, K. Kikuchi, H. Kokubun, Y. Kaizu, H. Kobayashi, J. Phys. Chem. 88, 1308 (1984). 